\title{
The Role of Chemotherapy in the Treatment of Kaposi's Sarcoma
}

\author{
I.Essadi ${ }^{1 *}$, Y.Sbitti ${ }^{1}$, M.Ichou $^{1}$ and H.Errihani ${ }^{2}$
}

${ }^{1}$ Medical Oncology Unit at Military Hospital Mohamed V-R abat

${ }^{2}$ Medical Oncology Unit at National Institute of Oncology-Rabat

\begin{abstract}
Kaposi's sarcoma (KS) is an opportunistic tumor proliferation Multicenter cells derived from lymphatic endothelium, infected with human herpes virus 8 (HHV-8). It is a heterogeneous disease distribution, which can be linked to poor prognosis. HHV-8 infection and immunosuppression are essential to its development $[1,2]$. These two elements are constant in the four clinical-epidemiological forms of KS: classical form, originally described by Moritz Kaposi typically affecting elderly men of Mediterranean, the endemic presence in Southern Africa, the epidemic form in patients infected HIV, and, finally, the MK complicating iatrogenic immunosuppression, particularly after organ transplantation [3]. This work exposes the real place of chemotherapy in adult patients with Kaposi sarcoma, among the available therapeutic means.
\end{abstract}

Keywords: Kaposi's sarcoma; Treatment; Chemotherapy

\section{Introduction}

Kaposi's sarcoma (KS) is a disease with heterogeneous distribution, affecting immunocompromised patients. Treatment depends on the particularities of each of the four clinical-epidemiological forms, but is based primarily on eliminating the factors maintaining immunosuppression [4]. Local treatments are proposed for lesions with a significant risk of recurrence, and had no effect on lesions which development in areas, is not covered [5]. Antiretroviral therapy should be initiated for all patients with HIV infection [4]. Chemotherapy will be given to disseminated forms, rapidly progressive, with visceral lifethreatening [5].

\section{Elements of Diagnosis}

\section{Clinic}

KS growths, called lesions or tumours, may be seen in a wide range of colours, from pink to red-violet to brown to blue.

Skin lesions: generally flat, painless and do not itch. They can look similar to a bruise. Do not blanch, or turn white, when pressure is applied. As the lesions progress, they may become elevated, can develop into nodules and can join together. Early on, these growths

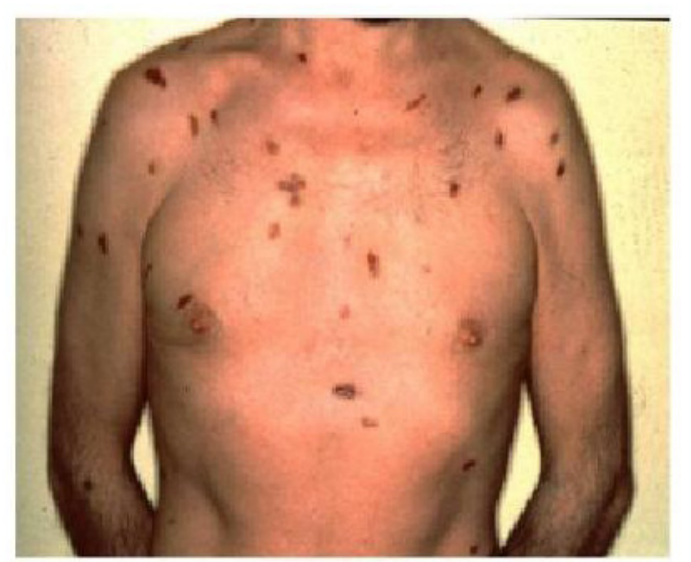

Figure 1:

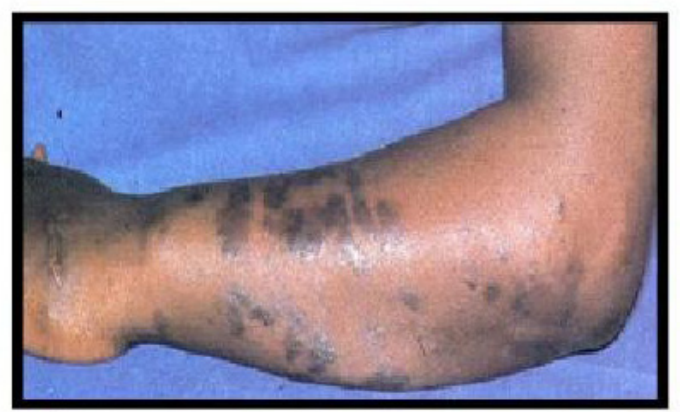

Figure 2:

may be very small and innocent looking, causing no problems except for concern about their appearance. The pace of the disease varies from person to person. In some, growth is slow with little change from month to month. In others, growth is rapid with new spots occurring almost every week (Figure 1 and 2).

The mucosal injury occurs less frequently characterized by compromised oral pharyngeal, anogenital or eye may ulcerate and secondary infection (Figure 3 and Figure 4) [6].

Lymph nodes: swelling of the feet, lower legs or genitals can occur as a result of the blocked lymph system. Occasionally swelling can occur around the eyes and face.

Lungs: recurrent chest infections, accumulation of fluid on the lungs and possibly blood in the spit, accompanied with coughing and breathlessness.

*Corresponding author: Dr. Essadi Ismail, Service d'oncologie médicale à l'hopital militaire d'instruction Mohamed V, 10100, Rabat, Maroc, Tel : (+212) 661168987; E-mail: ismail_onco@yahoo.fr

Received April 23, 2011; Accepted July 20, 2011; Published July 22, 2011

Citation: Essadi I, Sbitti Y, Ichou M, Errihani H (2011) The Role of Chemotherapy in the Treatment of Kaposi's Sarcoma. J Cancer Sci Ther 3: 145-000. doi:10.4172/1948-5956.1000077

Copyright: ( 2011 Essadi I, et al. This is an open-access article distributed under the terms of the Creative Commons Attribution License, which permits unrestricted use, distribution, and reproduction in any medium, provided the original author and source are credited. 
Citation: Essadi I, Sbitti Y, Ichou M, Errihani H (2011) The Role of Chemotherapy in the Treatment of Kaposi's Sarcoma. J Cancer Sci Ther 3: 145148. doi:10.4172/1948-5956.1000077

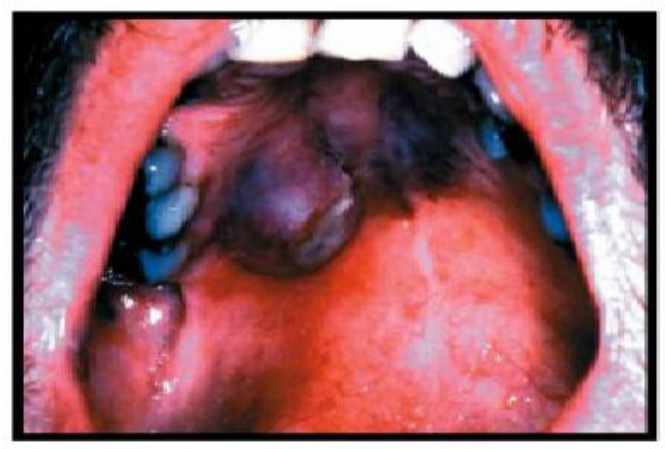

Figure 3:
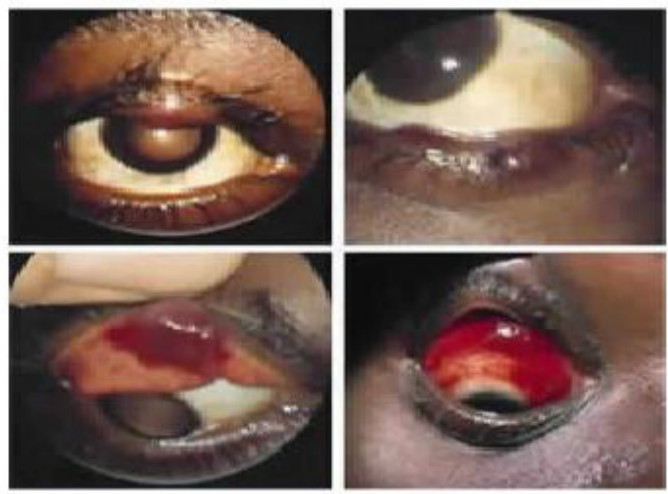

Figure 4:

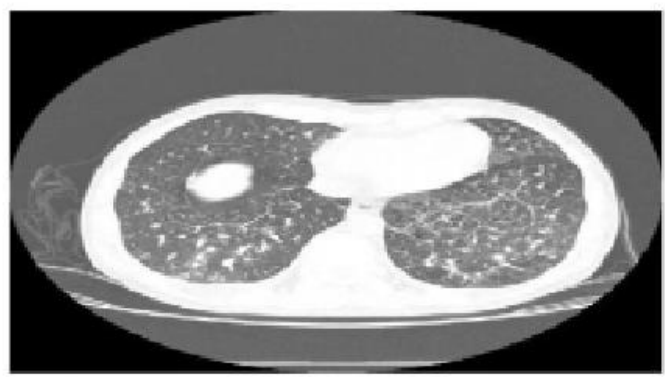

Figure 5:

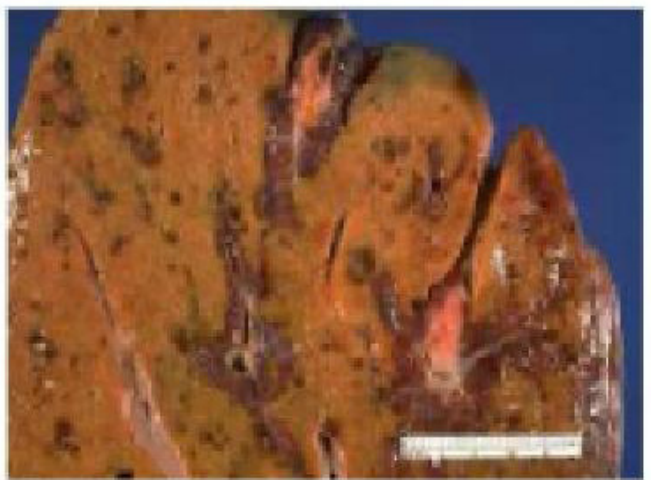

Figure 6:

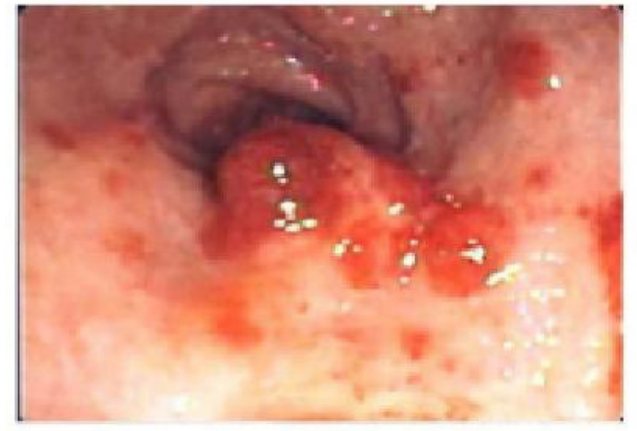

Figure 7:

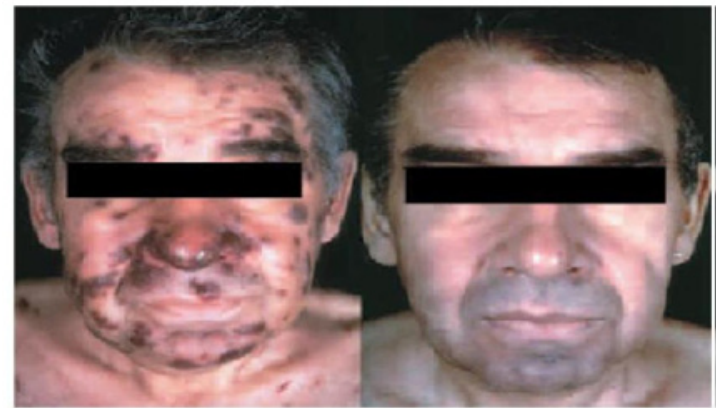

Figure 8:

Gastrointestinal tract: Can be visible in the mouth (as a skin lesion) or cause bleeding from the Gut. In fact, all organs may be affected (Figure 5, Figure 6 and Figure 7) [7].

Extension: Any patient in whom the diagnosis of KS was made will benefit from a thorough clinical examination, leading to a mapping of skin lesions and mucous membranes. Suspicion of a visceral realization will require further tests (CT scan, endoscopy and bronchoscopy with bronchoalveolar lavage) [7].

Classification and prognosis: Two prognostic classifications are available. The "TIS" classification described in 2003, is specific for HIV patients. It classifies the KS prognosis into two groups, based on the characteristics of the tumor (T) localized or widespread, the immunity (I) through the determination of CD4 T cells, and the systemic impact of the disease (S) (Table 1 data shown as Supplementary).

The second classification is specific to the classical form. It classifies patients into 4 stages depending on the extent and aggressiveness of skin involvement, speed of evolution and the complications it causes (Table 2 data shown as Supplementary).

The principles of treatment: In advanced forms, it is an incurable disease. The goal of treatment is purely palliative, aimed primarily at improving the quality of life by reducing symptoms and tumor size. The pathogenesis involves several factors, providing multiple therapeutic options available depending on the individual patient profile. Thus patients with HIV should receive antiretroviral therapy [8]. Among those who have undergone organ transplants, it must opt for a minimization of immune-deletion, which controls the Kaposi's proliferation [9]. KS on the skin is not in itself life threatening, and may resolve with HAART; this is the first live treatment. Larger lesions can 
also be treated with radiotherapy. If the KS has spread more widely, HAART may be sufficient or chemotherapy may be necessary as well $[8,9]$.

Role of chemotherapy: Current recommendations put chemotherapy in the heart of an aggressive therapeutic strategy, aimed at controlling disseminated forms with visceral involvement, and rapidly progressive or refractory to topical therapy [10,11]. Several clinical trials have evaluated the contribution of chemotherapy in the treatment of all clinical forms of KS. The results were promising, despite the small number of patients in most trials, which were mostly retrospective in nature [12]. Early studies had evaluated the time of poisons such as vinblastine and vincristine, alone or in combination with bleomycin, objective responses ranged between 10 and $75 \%$ [12]. A randomized study comparing etoposide is an inhibitor of the topoisomerase II with Vinblastine in the classical form. No significant difference was noted in terms of response and survival, but with greater hematologic toxicity in patients who received vinblastine [12]. Liposomal doxorubicin (LD) was evaluated first in patients with KS associated with HIV. An observational study of 54 patients, showed that the concomitant use of antiretroviral therapy and DL at a dose of $20 \mathrm{mg} / \mathrm{m}^{2}$ every two weeks, did not influence the rate of CD4 T lymphocytes, and gave encouraging responses (Figure 8) [13].

Three large randomized studies have followed the first, which compared the associations LD versus Bleomycin and Vinblastine plus or minus doxorubicin in first line. The objective responses were significantly better in patients who received the LD, with no impact on progression-free survival or overall survival, but with much less toxicity (Table 3 data shown as Supplementary) [14]. The LD has also demonstrated its effectiveness in the classical form of KS. Frontlines large multicenter retrospective study included 55 patients with advanced classic KS. These patients received the LD at a dose of $20 \mathrm{mg} /$ $\mathrm{m}^{2}$ every 3 weeks until progression or intolerable toxicity. The average objective response was $71 \%$ with a median response of 25 months and an acceptable toxicity profile by the authors [15]. Second row, a retrospective study evaluated the effectiveness of the LD in 35 patients with classic KS at an advanced stage. The average objective response was $80 \%$ with a progression-free survival of 9 months and 2 years overall survival of $75 \%$ [16]. Studies have evaluated the efficacy of taxanes in patients with visceral involvement of KS, pretreated by one or more lines of chemotherapy or immunotherapy. For patients without HIV, two studies have shown the efficacy of taxanes. The first study included 12 patients receiving paclitaxel or docetaxel in second line. All patients had responded to treatment, including six fully. No recurrence was recorded after a decline of 13 months [17]. The second Italian study, 17 patients or multiprocessing, have received weekly paclitaxel. The results were encouraging, since there were 10 complete responses, four partial responses, one up and two patients not evaluable at 12 weeks after treatment. The toxicity profile was dominated by sensory neuropathy, grade 1 or 2 without functional impairment [18]. For HIV patients, two phase 2 studies have been published. The patients received paclitaxel, after at least one prior therapy. The average objective answers were $71 \%$ and $56 \%$. The median response of 8.9 months and 10.4 months [19]. Other molecules have been evaluated mainly in patients pretreated. 12 patients with classic KS, received gemcitabine at a dose of $1.2 \mathrm{~g}$ every 2 weeks. The results were encouraging, with a complete response and 11 partial responses [20].

\section{Targeted therapies}

It appears that angiogenesis plays a role in lesion's development. Indeed, lesions of the KS consistently express VEGF C and the cells carry receptors to VEGF type 2 and 3. Other findings have resulted that the HHV-8 viral genome, play a role in the activation of VEGFR 2 and mediate the release of VEGF [21]. Several antiangiogenic agents are being evaluated. In the same sense, for the antiangiogenic properties of thalidomide, two studies have assessed its efficacy in advanced forms of KS. The results were controversial because of the toxicity of Thalidomide that caused the frequent stopping treatment [22]. Kaposi's lesions also express PDGFR and C-Kit. The targeting of these pathways has been evaluated in a study presented at ASCO 2004. Five patients received $300 \mathrm{mg}$ of Imatinib daily, with assessment of clinical response, pathological and biological after 4 weeks of treatment. The authors reported 3 partial responses and 2 stable diseases, with a pathological response in 3 patients and a biological response in 2 patients, 4 weeks after starting treatment [23]. These findings predict the role that will play targeted therapies in the treatment of $\mathrm{MK}$ in the years to come. Thus, several research protocols, attempting to assess the effect brought by blocking conduction pathways involved in cell proliferation cells, namely the mTOR pathway and PI3K [24].

\section{Conclusion}

KS is a disorder for which the pathogenesis is complex. The evolution of knowledge would reveal new therapeutic approaches. Chemotherapy is indicated in disseminated forms with visceral involvement. Targeted therapies offer an attractive and revolutionary therapeutic alternative. Prevention of viral infections, HIV and HHV-8 remains the only means of preventing the KS.

\section{References}

1. Antman K, Chang Y (2000) Kaposi's sarcoma. N Engl J Med 342: 1027-1038.

2. Chang Y, Cesarman E, Pessin MS, Lee F, Knowles DM, et al. (1994 Identification of herpesvirus-like DNA sequences in AIDS-associated Kaposi's sarcoma. Science 266: 1865-1869.

3. Lebbe C, Legendre C, Frances C (2008) Kaposi sarcoma in transplantation Transplant Rev (Orlando) 22: 252-261.

4. Cancer epidemiology unit, imperial cancer research fund (ICRF) Oxford UK (2000) Highly active antiretroviral therapy and incidence of cancer in human immunodeficiency virus-infected adults. J Natl Cancer Inst 92: 1823-1830.

5. Di Lorenzo G, Konstantinopoulos PA, Pantanowitz L, et al. (2007) Management of AIDS-related Kaposi's sarcoma. Lancet Oncol 8: 167-176.

6. Barete S, Calvez V, Mouquet C, Barrou B, Dantal J, et al. (2000) Clinica features and contribution of virological findings to the management of Kaposi sarcoma in organ-allograft recipients. Arch Dermatol 136: 1452-1458

7. Pellet C, Chevret S, Frances C, Euvrard S, Dalac S, et al. (2002) Prognostic value of quantitative Kaposi sarcoma-associated herpesvirus load in posttransplantation Kaposi sarcoma. J Infect Dis 186: 110-113.

8. Sullivan RJ, Pantanowitz L (2010) New drug targets in Kaposi sarcoma.Exper Opin Ther Targets 14: 1355-1366.

9. C. Francès, C. Lebbé La Lettre du Cancérologue $\bullet$ Vol. XIX - $\mathrm{n}^{\circ} 7$ - septembre 2010 La maladie de Kaposi

10. Bower M, Collins S, Cottrill C, Montoto S, Wales N, et al. (2008) British HIV Association guidelines for HIV-associated malignancies. HIV Med 9: 336-388.

11. Yarchoan R, Tosato G, Little RF (2005) Therapy insight: AIDS-related malignancies-the influence of antiviral therapy on pathogenesis and management. Nat Clin Pract Oncol 2: 406-415.

12. Di Lorenzo G (2008) Update on classic Kaposi sarcoma therapy: new look at an old disease. Crit Rev Oncol Hematol 68: 242-249.

13. Lichterfeld M, Qurishi N, Hoffmann C, Brockmayer NH, Mauss S, et al. (2005) Treatment of HIV-1-associated Kaposi's sarcoma with pegylated liposomal doxorubicin and HAART simultaneously induces effective tumor remission and CD4+ T cell recovery. javascript:AL get(this, 'jour', 'Infection.'); 33: 140-147. http://www.ncbi.nlm.nih.gov/pubmed/15940415. 
Citation: Essadi I, Sbitti Y, Ichou M, Errihani H (2011) The Role of Chemotherapy in the Treatment of Kaposi's Sarcoma. J Cancer Sci Ther 3: 145148. doi:10.4172/1948-5956.1000077

14. Pantanowitz L, Stebbing J, Dezube BJ (2010) Kaposi sarcoma. A model of oncogenesis (Textbook). Kerala, India. Research Signpost.

15. Di Lorenzo G, Kreuter A, Di Trolio R, Romano C, Bower M, et al. (2008) Activity and safety of pegylated liposomal doxorubicin as first-line therapy in the treatment of non-visceral classic Kaposi's sarcoma: a multicenter study. $J$ Invest Dermatol 128: 1578-1580.

16. Di Lorenzo G, Di Trolio R, Montesarchio V, Palmieri G, Nappa P, et al. (2008) Pegylated liposomal doxorubicin as second-line therapy in the treatment of patients with advanced classic Kaposi sarcoma: a retrospective study. Cancer 112: 147-152.

17. Fardet L, Stoebner PE, Bachelez H, Kerob D, Morel P, et al. (2006) Treatment with taxanes of refractory or life-threatening Kaposi sarcoma not associated with human immunodeficiency virus infection. Cancer 106: 1785-1789.

18. Brambilla L, Romanelli A, Bellinvia M, Ferrucci S, Vinci M, et al. (2008) Weekly paclitaxel for advanced aggressive classic Kaposi sarcoma: experience in 17 cases. Br J Dermatol 158: 1339-1344.

19. Phatak UA, Joshi R, Badakh DK, Gosavi VS, Jagdale RVet al. (2010) AIDS- associated cancers: an emerging challenge. J Assoc Physicians India 58: 159162.

20. Brambilla L, Labianca R, Ferrucci SM, Taglioni M, Boneschi V, et al. (2001) Treatment of classical Kaposi's sarcoma with gemcitabine. Dermatology 202 119-122.

21. Bais C, Van Geelen A, Eroles P, Mutlu A, Rafii S, et al. (2003) Kaposi's sarcoma associated herpesvirus $G$ protein-coupled receptor and activation of the VEGF receptor-2/ KDR. Cancer Cell 3: 131-143.

22. Ben M'barek L, Fardet L, Mebazaa A, Beit I, Morel P, et al. (2007) A retrospective analysis of thalidomide therapy in non-HIV-related Kaposi's sarcoma. Dermatology 215: 202-205.

23. Henry B Koon, Glenn J Bubley, Liron Pantanowitz, Smith B, Proper J, et al.(2005) Imatinib-Induced Regression of AIDS-Related Kaposi's Sarcoma. J Clin Oncol 23: 982-989.

24. Fabio Petrocca, Andrea Vecchione, and Carlo M. Croce (2008) Emerging Role of miR-106b-25/miR-17-92 Clusters in the Control of Transforming Growth Factor $\beta$ Signaling. Cancer Res 68: 8191-8194. 